\title{
Combining Shape and Position Expectancies: Hierarchical Processing and Selective Inhibition
}

\author{
Alan Kingstone \\ Age and Cognitive Performance Research Center, University \\ of Manchester, Manchester, England
}

\author{
Raymond Klein \\ Dalhousie University
}

\begin{abstract}
Two experiments report the effects of generating a concurrent position expectancy and form expectancy. Ss were precued to a stimulus position where 1 target shape was most probable, and they made a speeded 2-choice response to the orientation of the displayed shape. Response time (RT) was faster for an expected position than an unexpected position and faster for a likely shape than for an unlikely shape. This replicates the work of Lambert and Hockey (1986). It was also observed, however, that when a stimulus appeared at an unexpected position where 2 shapes were equally improbable, RT was slower for the shape that had been likely rather than unlikely at the cued position. This finding is incompatible with the probability-matching hypothesis of Lambert and Hockey. The data support a hierarchical-expectancy model of attentional selectivity.
\end{abstract}

Humans respond faster and/or more accurately to expected rather than unexpected visual events. This is true for expectancies involving stimulus shape (Posner \& Snyder, 1975), position (Posner, Snyder, \& Davidson, 1980), color (Humphreys, 1981), class (Neely, 1977), modality (Klein, 1977), and onset time (Klemmer, 1956).

To illustrate this general point, consider the study reported by Lambert and Hockey (1986, Experiment 2). Subjects performed a two-choice response time (RT) task in which they judged the orientation of a diamond- or elliptical-shaped stimulus presented $5^{\circ}$ to the left or right of central fixation. Subjects pressed one key if the stimulus was vertically oriented and another key if the stimulus was horizontally oriented. Because the orientation of a stimulus varied randomly from trial to trial, subjects never knew which response was required on any given trial. On one side of the display (e.g., the left), diamonds were always more likely than ellipses ( $p=.80$ vs. $p=.20$ ); on the other side (e.g., the right), ellipses were always more likely than diamonds ( $p=.80$ vs. $p=.20$ ). Before each trial, a location cue appeared at fixation, which indicated that the next stimulus was likely to appear on the left $(p=.80)$ and unlikely to appear on the right $(p=.20)$ or vice versa. Lambert and Hockey found that RT was faster when a stimulus appeared at the cued (expected) rather than uncued (unexpected) position. In addition, regardless of whether the

This research was conducted at the Age and Cognitive Performance Research Centre (ACPRC), University of Manchester, Manchester, England, and supported by Patrick Rabbitt and a Commonwealth Scholarship Award to Alan Kingstone by the Commonwealth Scholarship Commission in the United Kingdom. We thank Patrick Rabbitt and Elizabeth Maylor at the ACPRC; John Duncan; Glyn Humphreys; Tony Lambert; Peter Meudell; Arthur Samuel; and an anonymous reviewer for valuable comments on earlier versions of this article. We also thank Sal Connolly for translating the raw data in England.

Correspondence concerning this article should be addressed to Alan Kingstone, who is now at the Department of Psychology, Dalhousie University, Halifax, Nova Scotia, Canada B3H 4J1. stimulus position was cued or uncued, RT was faster for diamonds than ellipses if the stimulus appeared where diamonds were likely (e.g., on the left), and RT was faster for ellipses than diamonds if the stimulus appeared where ellipses were likely (e.g., on the right).

Lambert and Hockey (1986) suggested that their subjects generated and held in parallel multiple expectancies that reflected the precise long-term probability of a particular shape appearing at a particular position. This is called the probability-matching hypothesis. Although their results are consistent with this proposal, Lambert and Hockey's results are also consistent with the idea that a subject generates a single short-term shape expectancy appropriate for the cued location; if a stimulus appears at an unexpected position, the early processing of stimulus location enables a subject to select from memory an alternative shape expectancy. For example, if a stimulus is cued to appear on the left and diamonds are likely on the left, an individual may expect "a diamond on the left." If a stimulus appears unexpectedly on the right and ellipses are likely on the right, shape expectancy may change from a diamond to an ellipse. Here, a subject never actively holds more than one shape expectancy at any given time. This is called the hierarchical-expectancy hypothesis, which indicates that the early resolution of stimulus position determines whether a subject maintains or switches shape expectancy. There is ample behavioral (e.g., Garner, 1987; Posner et al., 1980, Experiment 2) and electrophysiological evidence (e.g., Harter, Aine, \& Schroeder, 1982; Hillyard \& Munte, 1984) suggesting that when a signal appears more than a few degrees outside of fixation in an otherwise blank visual field, stimulus position is resolved relatively more quickly than shape. Thus this alternative explanation must be considered a viable option. Lambert and Hockey acknowledged this possibility, and after two unsuccessful attempts to discriminate empirically between the proposals (see Experiments 3 and 4) they concluded that a test between the probabilitymatching hypothesis and the hierarchical-processing hypothesis "may prove extremely difficult to devise" (p. 494). This article presents such a test. 


\section{Experiment 1}

The design of our initial study was essentially the same as the Lambert and Hockey (1986) investigation except for two changes. First, instead of diamonds and ellipses as target stimuli, the letters $A$ and $V$ were used. Second, there were three target locations instead of two.

Subjects were required to perform a two-choice RT task in which they judged the orientation (upright/inverted) of the letter $A$ or the letter $V$. Either target stimulus could appear in the 4,8 , or 12 o'clock position of an imaginary clock face. The letter $A$ was likely at one position, and the letter $V$ was likely at another position. The major change from Lambert and Hockey's procedure was that the two letters were equally likely at the third position.

Both the probability-matching hypothesis and the hierarchical-expectancy hypothesis anticipate that as in the Lambert and Hockey investigation, regardless of whether a location is cued or uncued there is a performance advantage for the target shape that is likely at the location where stimulus onset occurs. For instance, if a target appears at the 4 o'clock position where the letter $A$ is more likely than the letter $V$, for example, then RT is faster for the letter $A$ than for the letter $V$. And if a target appears at the 8 o'clock location where the letter $V$ is more likely than the letter $A, \mathrm{RT}$ is faster for the letter $V$ than for the letter $A$.

But what will happen if subjects are cued to expect a target at the location where the letter $A$ is likely, for example, and a target unexpectedly appears at the location where the letters $A$ and $V$ are equally probable?

The prediction made by the probability-matching hypothesis is straightforward. Equiprobable stimuli are equally expected, and therefore there ought to be no preferential processing and no performance advantage for one target shape over the other regardless of whether the location is cued or uncued.

The outcome anticipated by the hierarchical-expectancy hypothesis is not as clear-cut. One possibility is that subjects will adopt a strategy of switching shape expectancies (e.g., from the letter $A$ to the letter $V$ ) when a position expectancy is disconfirmed, because the alternative shape (e.g., the letter $V$ ) is now, overall, more likely to occur. Thus, when target onset occurs at the uncued location where the letters $A$ and $V$ are equiprobable, RT is slower for the letter that was likely (e.g., the letter $A$ ) rather than unlikely (e.g., the letter $V$ ) at the cued position. A second possibility is that subjects will maintain rather than switch their initial shape expectancy: They correctly recognize that there is no a priori advantage in switching shape expectancies because both stimulus forms are now equally probable at the stimulated position. In this case we anticipate that at the uncued equiprobable-shape position, RT is faster for the letter shape that was likely (e.g., the letter $A$ ) rather than unlikely (e.g., the letter $V$ ) at the cued location. A third possibility is that subjects sometimes switch (and sometimes maintain) their initial shape expectancy, and these balance out so that there is no preferential processing and no performance advantage for one target shape over the other when presentation occurs at the uncued equiprobable-shape position. Although this proposal predicts the same mean RT as the probability hypothesis, it also predicts that the variance of the RT distribution at the equiprobable position depends on a mixture of RTs from when a shape expectancy is and is not switched.

\section{Method}

Subjects. Ten subjects ( 8 women and 2 men) from 18 to 26 years of age were recruited from the University of Manchester (Manchester, England). All reported normal or corrected-to-normal visual acuity. Each subject was paid $£ 2$ for participating in a 1-hr test session.

Apparatus and stimuli. Experimentation was conducted in a 13.4 square meter sound-damped room against a background of soft white noise. Two fluorescent ceiling lights provided ambient lighting. Subjects were tested individually in a two-choice RT task in which they judged the orientation (upright or upside down) of the letter $A$ or the letter $V$. These letters were chosen so that the stimulus shape ( $A$ or $V$ ) would need to be identified to determine orientation. With about a 50-cm viewing distance, subjects sat in front of an eye-level mediumresolution dark-screen color monitor connected to a BBC B+ microcomputer that controlled stimulus presentation and response tabulation. All stimulus items were white and presented on a black background. Two keys on the bottom row of the computer keyboard (" $\mathrm{Z}$ " and " $/ "$ ) were used as response keys. Subjects were instructed to respond as quickly and as accurately as possible by pressing either the left or right key with the index finger of the left or right hand, respectively. If at any time subjects accidentally pushed any key other than a response key, the computer beeped, which indicated that they should make sure that their fingers were resting lightly on the appropriate keys.

Materials were drawn from a three-item population: $A, V$, and an arrow. From a viewing distance of $50 \mathrm{~cm}$, each letter subtended a visual angle of $0.69^{\circ}$ in height and $0.57^{\circ}$ in width; the length of the arrow and width of the arrowhead subtended $0.69^{\circ}$ and $0.46^{\circ}$, respectively.

Procedure. Each trial began with the presentation of a central arrow cue that indicated the likely location of the target stimulus. After $1,500 \mathrm{~ms}$, the target (the letter $A$ or the letter $V$ ) appeared $4^{\circ}$ from the cue. The cue-target visual angle measures the distance from the center of the cue to the center of the target. The target appeared at 4,8 , or 12 o'clock of an imaginary clock face surrounding the cue. The letter target could be upright or upside down. Target orientation was randomly determined. Subjects pressed one response key if a target was upright and another response key if a target was upside down. Half of the subjects used the left finger to indicate upright and the right finger to indicate inverted; the other half performed conversely. Response execution terminated the cue and target and triggered an intertrial interval that varied randomly between 800 and $1,200 \mathrm{~ms}$.

Location cues were arrows that pointed at 4,8 , or 12 o'clock. Cues were equally probable and randomly selected. Each location cue pointed toward the location where the target onset was likely ( $p=$ $.80)$ and away from the two positions where target onset was unlikely ( $p=.10$ for each location).

At one position the letter $A$ was consistently more likely to occur than the letter $V(p=.80$ vs. $p=.20)$, at a second location the letter $V$ was consistently more likely to occur than the letter $A(p=.80$ vs. $p=.20$ ), and at a third neutral-shape position the letters $A$ and $V$ were equally probable $(p=.50)$.

Instructions. In Experiment 1 and Experiment 2, three points were particularly emphasized during instruction. First, subjects must fixate on and must not remove their eyes from the central warning cue. Second, on every trial subjects ought to try to use the information provided by the cue to prepare for the target stimulus. That is, they were asked to attend to the cue and to use it rather than to accept the passive option of ignoring it entirely and attending only to the 
response stimulus. Third, subjects ought to respond as quickly and as accurately as possible. Subjects were told the significance of the location cue and the probability of a particular shape appearing at a particular position. To ensure that subjects understood the probability manipulations, they were quizzed informally by the experimenter. For example: "What does an arrow mean?" "What letter is likely at each position?" "What is the chance of receiving a letter at the 12 o'clock position if the arrow is pointing at 4 o'clock?" "What letter is

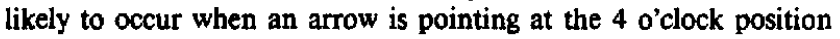
but a shape appears at the 8 o'clock location?"

Design. Experimental sessions were run in blocks of 100 trials. A single practice block always preceded a series of test blocks. A block of trials started with the instruction on the screen "PUSH SPACE BAR TO BEGIN BLOCK." When subjects felt ready to begin, they pressed the space bar and the screen went blank. Three s later, the first central cue of the first trial appeared on the screen. At the end of a block (about 5-8 min), the message "RECORDING DATA" appeared on the screen (approximately $15 \mathrm{~s}$ ) followed by the space bar instructions which signaled that the next block was ready to begin.

Subjects received one block of practice trails followed by eight blocks of test trials. Test trials were composed of 640 cued-location trials (valid location cue) and 160 uncued-location trials (invalid location cue). Similarly, these 800 test trials were composed of 426 likely shape trials (the target shape was probable for the location where stimulus onset occurred), 267 neutral-shape trials (the target shapes were equally probable for the location where stimulus onset occurred), and 107 unlikely shape trials (the target shape was improbable for the location where stimulus onset occurred). Location and shape conditions were combined orthogonally.

For 5 subjects the letter $A$ was likely at the 4 o'clock position, the letter $V$ was likely for the 8 o'clock location, and the letters $A$ and $V$ were equally probable at the 12 o'clock position. For the other 5 subjects, the letter $V$ was likely at the 4 o'clock position, the letter $A$ was likely at the 8 o'clock location, and the letters $A$ and $V$ were equally probable at the 12 o'clock position. Pilot research found that when the neutral- (equiprobable-) shape location was positioned at either the 4 o'clock or 8 o'clock position, subjects tended to treat the combined 4 and 8 o'clock display positions simply as one "bottom" location-where either the letter $V$ or the letter $A$ was most probable overall. For example, if the neutral- (equiprobable-) shape location was positioned at the 80 'clock position and the letter $A$ was likely at the 4 o'clock position, subjects tended to treat the 4 and 8 o'clock locations as one spatial region, for example, "the bottom of the display where the letter $A$ is most probable overall." The present design avoided this problem by placing the neutral-shape location at the peak 12 o'clock display position at all times.

\section{Results}

In Experiments 1 and 2, practice data were not recorded or analyzed. Error trials and correct response trials were identified. The first trial after an error was discarded from analysis to reduce the variability produced by longer RTs, which follow response errors (Rabbitt, 1966). From the remaining trials, mean correct RTs were calculated for each subject and each condition. Separate analyses of variance (ANOVAs) were conducted on the error rate and correct RT data.

Separate three-way ANOVAs were conducted on the mean correct RT and error data, with location (cued or uncued), shape (likely, neutral, or unlikely), and orientation (upright or inverted) as within-subject variables. Mean correct RTs and error percentages are shown in Table 1 as a function of target location and target shape.

To avoid any confusion in data interpretation, it is important that we establish clearly what is meant by a likely, neutral,
Table 1

Mean Correct Response Times (RTs) and Error Percentages for Likely, Neutral, and Unlikely Shapes at Cued and Uncued Locations

\begin{tabular}{cccccc}
\hline & \multicolumn{2}{c}{ Cued location } & & \multicolumn{2}{l}{ Uncued location } \\
\cline { 2 - 3 } \cline { 5 - 6 } Shape & $\begin{array}{c}\text { Error } \\
\text { percentage }\end{array}$ & RT & & $\begin{array}{c}\text { Error } \\
\text { percentage }\end{array}$ & RT \\
\hline Likely & 4.0 & 573 & & 6.2 & 714 \\
Neutral & 5.9 & 599 & & $6.0^{\circ}$ & $772^{2}$ \\
Unlikely & 5.7 & 641 & & 5.9 & 786 \\
\hline
\end{tabular}

Note. All times are in milliseconds.

-At the cued location, target shape was likely(error percentage $=6.2$, $\mathrm{RT}=792)$ /unlikely $($ error percentage $=5.8, \mathrm{RT}=752$ ).

or unlikely shape at a cued or uncued location. Assume that the letter $A$ is likely at the 4 o'clock position, the letter $V$ is likely at the $8 o^{\prime}$ 'clock position, and the letters $A$ and $V$ are equiprobable at the 12 o'clock position. If the 12 o'clock position is cued and the target occurs at the 12 o'clock position, then this is a cued-location neutral-shape trial. For instance, if the 4 o'clock (letter $A$ ) position is cued and a target appears at the $\mathbf{4}$ o'clock location, this is a cued-location likely shape trial if the target shape is the letter $A$ and a cuedlocation unlikely shape trial if the target shape is the letter $V$. Conversely, if the 4 o'clock (letter $A$ ) position is cued (or the 12 o'clock neutral position is cued) and a target appears at the uncued 8 o'clock (letter $V$ ) position, this is an uncuedlocation likely shape trial if the target shape is the letter $V$ and an uncued-location unlikely shape trial if the target shape is the letter $A$.

RT analysis revealed significant main effects for location, $F(1,9)=24.70, p<.01$; shape, $F(2,18)=7.68, p<.01$; and orientation, $F(1,9)=17.44, p<.01$. Response times were, respectively, 604 and $757 \mathrm{~ms}$ for cued and uncued locations; 644,686 , and $714 \mathrm{~ms}$ for likely, neutral, and unlikely shapes; and 655 and $706 \mathrm{~ms}$ for upright and inverted targets. No interaction approached significance.

These findings suggest that whether a position is cued or uncued, there is a performance advantage for the shape that is probable at the location where the target appears. For example, assume that the letter $A$ is likely at the 4 o'clock position, the letter $V$ is likely at the 8 o'clock position, and the letters $A$ and $V$ are equiprobable at the $120^{\circ}$ clock position.

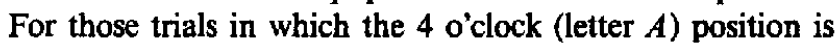
cued and a stimulus appears at that location, RT is faster for the letter $A$ than for the letter $V$; but on those trials in which the 4 o'clock (letter $A$ ) location is cued and a stimulus appears at the $8 o^{\prime}$ 'clock (letter $V$ ) position, RT is faster for the letter $V$ than for the letter $A .^{\prime}$ These data replicate the standard finding of Lambert and Hockey (1986).

\footnotetext{
'Table 1 shows that the difference between a likely and an unlikely shape at an uncued position is $72 \mathrm{~ms}(714 \mathrm{~ms}$ vs. $786 \mathrm{~ms})$. This difference reflects a combination of trials when the cued location was either the 12 o'clock neutral-shape position or the $40^{\prime}$ clock $/ 8$ o'clock likely shape position. In the first case the difference between a likely and unlikely shape at an uncued position was $66 \mathrm{~ms}(730 \mathrm{~ms}$ vs. 796 $\mathrm{ms}$ ), and in the second case the difference was $78 \mathrm{~ms}$ (698 ms vs. 776 $\mathrm{ms}), F(1,27)<1.00$.
} 
The probability-matching hypothesis predicts no difference between equiprobable shapes when a position expectancy is disconfirmed. From Table 1 we see that this null result was not supported: At the uncued neutral- (equiprobable-) shape location, RT was slower for the shape that had been likely rather than unlikely at the cued location (792 ms vs. $752 \mathrm{~ms}$ ), $F(1,9)=7.73, p<.05$. To illustrate this finding, we assume again that the letter $A$ is likely at the 4 o'clock position, the letter $V$ is likely at the $8 o^{\prime}$ clock position, and the letters $A$ and $V$ are equiprobable at the 12 o'clock position. When the 4 o'clock (letter $A$ ) position is cued and a stimulus appears unexpectedly at the 12 o'clock (neutral-shape) location, RT performance at the uncued 12 o'clock location is slower for the letter $A$ than for the letter $V$. Similarly, when the 8 o'clock (letter $V$ ) location is cued but target onset occurs at the uncued neutral-shape 12 o'clock location, RT is slower for the letter $V$ than for the letter $A$.

Error analysis failed to show any significant differences between conditions. The overall error rate was $5.6 \%$.

\section{Discussion}

Response time is faster at cued positions than at uncued positions. At the cued location, RT is shortest for the shape that is likely there; at an uncued location RT is shortest for the shape that is actually most probable at this unexpected position. Thus, regardless of whether a position is cued or uncued, there is a performance advantage for the shape that is likely for that position; that is, RT is faster for the letter $A$ than for the letter $V$ when a stimulus appears at the location where the letter $A$ is likely, and RT is faster for the letter $V$ than for the letter $A$ when target onset occurs at the location where the letter $V$ is likely. These data replicate those of Lambert and Hockey (1986), and as noted previously, they agree with either the hierarchical-expectancy hypothesis or the probability-matching hypothesis.

Nevertheless, our finding that RT is significantly slower at the uncued equiprobable-shape position for the shape that had been likely rather than unlikely at the cued position clearly disagrees with the probability-matching hypothesis of Lambert and Hockey (see also Lambert, 1987). According to this model, equiprobable shapes are equally expected regardless of whether a position expectancy is confirmed or disconfirmed. Thus, in Experiment 1 performance latencies ought to have been the same for equiprobable shapes when a position expectancy was disconfirmed. This was clearly not the case.

How might the hierarchical-processing hypothesis account for the finding that RT was $40 \mathrm{~ms}$ slower for the shape that had been likely rather than unlikely at the cued position? The obvious possibility is that when a position expectancy is disconfirmed, subjects switch shape expectancies. This is a reasonable strategy for subjects to adopt because when a position expectancy is disconfirmed, the alternative shape is now overall more likely to occur.

There is a problem with this proposal, however. The disadvantage for the cued-location shape is roughly twice as large when it is presented at an uncued position where it is unlikely ( $78 \mathrm{~ms}$; see Footnote 1) as compared with an uncued position where it has a $50 \%$ chance of being presented $(40 \mathrm{~ms})$, a difference that 9 out of 10 subjects showed ( $p<.025$, according to a two-tailed sign test). ${ }^{2}$ If subjects switch shape expectancies in both cases, why is the disadvantage for the cuedlocation shape stronger at the location where the other shape is most probable than at the equiprobable-shape position?

Selective inhibition of the cued-location shape may provide an explanation. Kingstone (in press) has observed that when target position is resolved relatively more quickly than a target shape, disconfirmation of a position expectancy may lead to slower processing of the shape that had been likely at the cued position-wherever it appears. Subject's become "suspicious" of the shape that was likely at the cued location and take longer to evaluate it when a position expectancy is disconfirmed. Such a selective-inhibition effect could account for our observation that there is a $40-\mathrm{ms}$ RT disadvantage of the cued-location shape when it appears at the uncued equiprobable-shape position. It is not that subjects are facilitated in processing the shape that was unlikely at the cued position; rather, subjects are selectively inhibited in processing the shape that was likely at the cued location. In other words, subjects cancel (i.e., selectively inhibit) the original shape expectancy when targets are unexpectedly discovered to be equiprobable. This selective inhibition is revealed as a $40-\mathrm{ms}$ RT disadvantage of the cued-location shape at the uncued equiprobable-shape position. Subjects do not switch their shape expectancy; they merely inhibit the original one.

The implication of this proposal is that when a position expectancy is disconfirmed and subjects do switch shape expectancies, there ought to be a selective inhibitory effect in processing the shape that was likely at the cued position (and is now unlikely at the uncued position) and a facilitatory effect in processing the shape that was unlikely at the cued position (and is now likely at the uncued position). Facilitation of the shape that is probable at the stimulated (uncued) position as well as inhibition of the shape that was likely at the cued position agrees with our finding that the disadvantage of the cued-location shape is almost twice as large at the uncued position where the other shape is most probable (78 $\mathrm{ms})$ than at the neutral-shape position $(40 \mathrm{~ms})$.

It is important to note that switching shape expectancies from Shape 1 to Shape 2 and selective inhibition of Shape 1 are not mutually exclusive within the context of the hierarchical-processing hypothesis. Indeed, we believe that both mechanisms may be operating in Experiment 1. In the switching situation, the processing efficiency of Shape 2 is improved (a facilitation effect) and the processing efficiency of Shape 1 is reduced (an inhibition effect); with selective inhibition, only the processing efficiency of Shape 1 is reduced (an inhibition effect). In both cases, however, a single shape expectancy is subservient to the earlier resolution of a position expectancy,

\footnotetext{
${ }^{2}$ When this difference was evaluated by computing a planned contrast or by the interaction term in an ANOVA, it was only marginally significant; however, this was due to only 1 aberrant subject. Because 9 out of 10 subjects show this trend, and it is highly significant when the stray subject is excluded, $F(1,24)=7.88, p<$ .01 , we are confident that it represents a real difference.
} 
that is, the position expectancy-shape expectancy relation is a hierarchical one. Disconfirmation of a position expectancy delays RT to the associated, expected Shape 1 wherever it appears and triggers the generation of a new shape expectancy if the rapid analysis of target location information reveals that the stimulated position is one in which Shape 2 is now more likely than Shape 1.

In summary, we confirmed the standard finding of Lambert and Hockey (1986) that RT is faster for the probable shape at the stimulated position regardless of whether the position is cued or uncued. But we also found that at an uncued neutral position, where shapes were equally probable, RT is slower for the shape that is likely rather than unlikely at the cued location. This critical result disconfirms the probabilitymatching hypothesis. It is, however, compatible with two accounts of the hierarchical-expectancy hypothesis. One possibility is that shape expectancies are switched when a position expectancy is disconfirmed. A second possibility is that the original shape expectancy is selectively inhibited when a position expectancy is disconfirmed. We emphasized that switching shape expectancies and selectively inhibiting an original shape expectancy are not mutually exclusive within the context of the hierarchical-expectancy hypothesis.

Experiment 2 was designed with three goals in mind: (a) to replicate our key finding that at an uncued position where two shapes are equiprobable, RT is longest for the shape that was probable at the cued location; (b) to determine whether this effect is best viewed as a product of expectancy switching or selective inhibition; (c) to seek converging evidence for our proposal that expectancy switching and selective inhibition can operate in concert.

\section{Experiment 2}

In this second experiment the task was arranged along the lines of Experiment 1, with one important modification. There were now three target stimuli $(A, V$, and $G)$, each of which tended to occur at a particular spatial location. For example, the letter $A$ was likely (with the letters $V$ and $G$ equiprobable) at the 40 'clock position; the letter $V$ was likely (with the letters $G$ and $A$ equiprobable) at the 8 o'clock position; and the letter $G$ was likely (with the letters $A$ and $V$ equiprobable) at the 12 o'clock position. Response choice was again based on target orientation. The letter $G$ was chosen as the third target shape because it shared both the open gap common to the letters $A$ and $V$ and the horizontal bar that was a distinguishing feature of the letter $A$. Note that the back of the letter $G$ was straight and not curved.

On the basis of Experiment 1 and the Lambert and Hockey (1986) investigation, we predicted that if a target appears at the 4 o'clock position where the letter $A$ is likely, for example, then RT is faster for the letter $A$ than for the letters $V$ and $G$. If a target appears at the 8 o'clock position where the letter $V$ is likely, for example, or at the 12 o'clock (letter $G$ ) position, latencies are shortest for the letter $V$ or the letter $G$, respectively. According to the probability-matching hypothesis, this data pattern occurs because subjects generate and hold in parallel long-term multiple shape-position expectancies, "accurately reflecting the statistical structure of the display"
(Lambert and Hockey, 1986, p. 494). According to the hierarchical-processing hypothesis, the data pattern occurs because the rapid resolution of target location information cues subjects either to maintain or to switch their single short-term expectancy for the target shape that is likely at the stimulated position. For example, if the 4 o'clock (letter $A$ ) position is cued, subjects generate an expectancy for the letter $A$ at the 4 o'clock position. If a stimulus appears at the 4 o'clock position as expected, subjects maintain their (letter $A$ ) shape expectancy. If the 4 o'clock (letter $A$ ) is cued, however, and a target unexpectedly appears at the uncued 12 o'clock (letter $G$ ) position, the early processing of target location information enables subjects to switch their shape expectancy from the letter $A$ to the letter $G$. Thus RT is faster for the letter $G$ than for the letters $A$ or $V$ at the uncued 12 o'clock (letter $G$ ) position.

The crucial question is this: In this example, will there be any RT difference between the unlikely equiprobable letters $A$ and $V$ ? More to the point, will RT be slower for the letter $A$ than for the letter $V$ when an expected (cued) shape (the letter $A$ ) appears at an uncued location? Only the selectiveinhibition mechanism of the hierarchical-processing hypothesis explains such an effect. It cannot be attributed to switching shape expectancies because subjects are assumed to change their expectancy to the letter $G$ and not to the letter $V$. And it cannot be explained by Lambert and Hockey's (1986) probability-matching hypothesis because by hypothesis equiprobable forms are equally expected, and thus there ought to be no performance advantage for one target shape over another.

\section{Method}

Subjects. Ten subjects ( 8 women and 2 men) from 18 to 23 years of age, 9 of whom had served in the preliminary investigation, were recruited as before.

Task. As in Experiment 1, subjects were required to judge letter orientation in a two-choice RT task; the testing room, the apparatus, the viewing distance, the size of the stimuli, the length of stimulus sequences, and the method of experimental instruction, target presentation, and response execution remained unchanged. Unlike Experiment 1 , there were three letter targets: $A, V$, and $G$. At one position the letter $A$ was more likely to occur than the letters $V$ or $G$ ( $p=.80$ vs. $p=.10$ for each of the two unlikely letters), at a second location the letter $V$ was more likely than the letters $A$ or $G(p=.10$ for each unlikely item), and at a third position the letter $G$ was more likely than the letters $A$ or $V(p=.80$ vs. $p=.10$ for each unlikely item).

Design. Subjects received one block of 100 practice trials, followed by eight blocks of 100 test trials. Test trials were composed of 640 cued-location trials (valid location cue) and 160 uncued-location trials (invalid location cue). Similarly, there were 640 likely shape trials (the target shape was probable for the position where stimulus onset occurred) and 160 unlikely shape trials (the target shape was improbable for the position where stimulus onset occurred). Location and shape conditions were combined orthogonally.

For 3 subjects the letter $A$ was likely at the 4 o'clock location, the letter $V$ was likely for the 8 o'clock location, and the letter $G$ was likely at the 12 o'clock location; for 3 other subjects it was $G$ at 4 o'clock, $A$ at $8 o^{\prime}$ 'clock, and $V$ at 12 o'clock; for the remaining 4 subjects it was $V$ at $4 o^{\prime}$ clock, $G$ at 8 o'clock, and $A$ at 12 o'clock. 
Table 2

Mean Correct Response Times (RTs) and Error Percentages for Likely and Unlikely Shapes at Cued and Uncued Locations

\begin{tabular}{cccccc}
\hline & \multicolumn{2}{c}{ Cued location } & & \multicolumn{2}{c}{ Uncued location } \\
\cline { 2 - 3 } \cline { 5 - 6 } Shape & $\begin{array}{c}\text { Error } \\
\text { percentage }\end{array}$ & RT & & $\begin{array}{c}\text { Error } \\
\text { percentage }\end{array}$ & RT \\
\hline Likely & 3.8 & 582 & & 4.3 & 713 \\
Unlikely & 5.2 & 645 & & $4.1^{2}$ & $777^{2}$ \\
\hline
\end{tabular}

Note. All times are in milliseconds.

at the cued location, target shape was likely(error percentage $=4.2$, $\mathrm{RT}=796) /$ unlikely(error percentage $=4.0, \mathrm{RT}=757$ ).

\section{Results}

Separate three-way ANOVAs were conducted on the mean correct RT and error data, with location (cued or uncued), shape (likely or unlikely), and orientation (upright or inverted) as within-subject variables. Mean correct RTs and error percentages are shown in Table 2 as a function of target location (cued or uncued) and target shape (likely or unlikely).

RT analysis revealed significant main effects for location, $F(1,9)=64.56, p<.01$; shape, $F(1,9)=13.74, p<.01$; and orientation, $F(1,9)=15.57, p<.01$. Response times were, respectively, 614 and $745 \mathrm{~ms}$ for cued and uncued locations; 648 and $711 \mathrm{~ms}$ for likely and unlikely shapes; and 652 and $706 \mathrm{~ms}$ for upright and inverted targets. No interaction approached significance. These findings indicated that whether a position is cued or uncued, there is a processing advantage for the shape that is probable at the location where the target appears.

Is there is a disadvantage for the cued-location shape when it appears at an uncued position in relation to an equiprobable control shape? For example, if the cued location is where the letter $A$ is probable and a target appears at the uncued location where the letter $G$ is probable, and the shape is not the letter $G$, is RT slower for the letter $A$ than for the letter $V$ even though $A$ and $V$ are equiprobable? Data analysis found that RT was indeed slower for the cued-location shape at an uncued position in relation to its equiprobable control (796 ms vs. $757 \mathrm{~ms}), F(1,9)=5.86, p<.05 .^{3}$

Error analysis failed to reveal any significant differences between conditions. The overall error rate was $4.3 \%$.

\section{Discussion}

The data show that RT is faster at cued positions than uncued positions. And regardless of whether a location is cued or uncued, RT is faster for the shape that is likely at that position. These findings replicate those of Lambert and Hockey (1986) and Experiment 1 and are consistent with either the probability-matching hypothesis or the switching aspect of the hierarchical-expectancy hypothesis.

The critical result, however, is the unequivocal finding of selective inhibition, that is, an RT difference between two equally improbable shapes at an unexpected position: RT was significantly slower for the shape that had been likely rather than unlikely at the cued position. This RT difference cannot be explained by the Lambert and Hockey (1986) probabilitymatching hypothesis (because the shapes are equiprobable). It is, however, readily compatible with the proposal of the hierarchical-expectancy hypothesis that selective inhibition of a shape expectancy associated with the cued target location may occur when a position expectancy is disconfirmed.

In summary, the data support a hierarchical-processing model of attentional selectivity. This assumes that when the processing of one stimulus attribute (e.g., location) is sufficiently faster than the processing of another (e.g., shape), the output derived from the earlier resolved attribute may "feed forward" and affect response performance for the other analyzed attribute. If a stimulus appears at an unexpected position where one shape is more likely than another, subjects inhibit the previous shape expectancy and switch to the alternative likely shape. When shapes are equiprobable at an unexpected position, inhibition of the previously held shape expectancy is revealed as a special delay in RT performance for the shape that was likely at the cued (expected) position. This model has recently been advanced by Kingstone (in press) and it assumes a parallel flow of different information from a single stimulus source, which is an assumption shared by many recent attentional theories such as the continuous-flow model of Eriksen and Schultz (1979), the cascade model of McClelland (1979), the isolable-systems model of Posner (1978), and the guided search model of Cave and Wolfe (1990).

We suggest that feed/forward relation, which gives rise to the inhibition phenomenon, is tied to the operation of a limited-capacity mechanism. For instance, when a position expectancy is disconfirmed, attention is withdrawn from the shape expectancy, causing the originally expected shape to suffer from slower processing in relation to other stimuli. This inhibition of a previously held shape expectancy is reminiscent of the "inhibition of return" phenomenon reported by Posner (e.g., Posner \& Cohen, 1984). With inhibition of return, if attention is drawn away from a previously attended or stimulated position, subjects are slower to process and respond to a target if it appeared there rather than elsewhere in the visual field (see also Maylor and Hockey, 1985; Rafal, Calabresi, Brennan, \& Sciolto, 1989). This correspondence with the inhibition-of-return phenomenon may be only superficial, however, for when (as in the present study) central cues are used to generate a location expectancy, withdrawing attention from a cued location does not result in inhibition of return (Posner \& Cohen, 1984; Rafal et al., 1989). It is therefore perhaps more appropriate to speculate that the present selective-inhibition effect draws on inhibitory processes similar to those involved in negative priming (e.g., Neill, 1977; Tipper, 1985; Tipper \& Cranston, 1985). With negative priming, if attention is first shifted away from an object

\footnotetext{
${ }^{3}$ It has been suggested that the selective-inhibition effect observed here may be unique to situations in which the target stimuli, $A$ and $V$, are highly confusable. This is not the case: Inhibition occurs for all shapes.
} 
representation, then RT is delayed when the ensuing target is the same or categorically related to the previously "ignored" object. This corresponds closely with our suggestion that the selective-inhibition effect may be characterized as an active negative shift in attention away from an initial shape representation-be it by canceling an original shape expectancy or by switching attention to a new shape expectancy. A third possibility is that the selective-inhibition effect does not involve the operation of a limited-capacity mechanism, and hence it is neither the perceptual processing of shape information that is inhibited nor the processes that operate between perception and response. Instead, selective inhibition may be characterized as a conservative criterion shift; that is, subjects are reluctant to initiate a response associated with the original shape expectancy when the contingent position expectancy is disconfirmed. Note, though, that our failure to observe evidence of a speed-accuracy tradeoff argues against this latter possibility. Regardless of the precise mechanisms that generate the selective-inhibition effect, however, the major finding is that when a stimulus appears at an unexpected position where two shapes are equally improbable, RT is slowest for the shape that was first expected at the cued position.

This result may be restated as suggesting that if a position expectancy is disconfirmed, then performance is enhanced if a shape expectancy is disconfirmed as well. Perhaps the selective-inhibition effect reflects a bias for congruence between a position expectancy and a shape expectancy within a single trial? Previous research suggests that subjects are often biased toward a global consistency of responses, events, and decisions, both across trials and within a single trial. For example, take the work of Williams (1966) and Rabbitt and Vyas (1974), who reported that in a same-different two-choice RT task, subjects are especially slow to implement a different judgment if they must repeat the response from the previous trial (i.e., the response sequence on trial $n-1$ and trial $n$ is different-different). Similarly, subjects are unusually slow to implement a same judgment if they must switch the response from the previous trial (i.e., the response sequence on trial $n$ -1 and trial $n$ is different-same). In a related vein, Fletcher and Rabbitt (1978) and Krueger and Shapiro (1981) hypothesized that when making same-different judgments, subjects tend to use an intertrial "bypass rule": Switch responses unless some aspect of the stimulus on the preceding trial is repeated. In terms of intratrial effects, Hedge and Marsh (1975) showed that the traditional Simon effect (faster responding to a symbolic stimulus that calls for a left response if it appears on the left rather than the right, and vice versa) is reversed if a symbolic stimulus-response mapping is incompatible (see also Stoffels, van der Molen, \& Keuss, 1985, 1989). For example, if subjects are instructed to push a red response key on their left when a green stimulus appears and a green response key on their right when a red stimulus appears, subjects are faster to respond to a green stimulus that appears on the right than on the left and faster to respond to a red stimulus on the left than on the right.

According to the global consistency account, the selectiveinhibition effect occurs when a position and shape expectancy are incongruent. According to the hierarchical-expectancy hypothesis, this is a necessary but not sufficient condition.
Fundamental to the expectancy hypothesis is the notion that there is a temporal hierarchical-processing relation between stimulus attributes, in which the early resolution of one attribute expectancy (e.g., location) influences the processing of another attribute expectancy (e.g., shape). In other words, the proposal is that target position and target shape are processed simultaneously, and in the present experiments, over different time scales: Target position is resolved more rapidly than target shape, hence the confirmation or disconfirmation of the position expectancy influences the attentional weight placed on a shape expectancy. In support of this proposal, Kingstone (in press) reports that an RT inhibition effect between mismatching position and shape expectancies can be abolished by reducing target position discriminability so that target position is resolved at about the same time as target shape. This finding disconfirms the global consistency account and converges with the hierarchical-expectancy hypothesis.

In the Kingstone (in press) study, subjects were required to judge the orientation of the letter $A$ or the letter $V$. On each trial subjects received a central cue indicating both where a target was likely to appear (to the left or right of fixation) and what the target shape was likely to be (the letter $A$ or $V$ ). Results showed that when target position was resolved more rapidly than target shape, RTs were unusually slow if target position and target shape expectancies were incongruent; that is, RT was delayed especially when an unexpected (uncued) shape appeared at an expected (cued) position or an expected (cued) shape appeared at an unexpected (uncued) position. This interaction between mismatching position and shape expectancies was eliminated, however, when target position discriminability was made difficult (by placing a distractor item [the letter $O$ ] in the location not containing a target) so that target position and target shape were resolved at about the same time. In other words, if the resolution of target position and target shape was approximately concurrent, then position and shape expectancies did not interact. In a separate experiment, it was observed that when target position is processed more quickly than target shape, an interaction between position and shape expectancies is influenced more by manipulating the validity of the position cues than the shape cues. This finding provides another piece of converging evidence in favor of the hierarchical-processing notion that the more rapidly resolved expectancy (in this case, target position rather than target shape) is the controlling factor of an RT inhibition effect. Finally, Kingstone (in press) reports that the inhibition effect is not unique to interactions between position and shape expectancies. Inhibition was also observed between expectancies for target color and target shape as well as between expectancies for target onset time and target shape.

One might ask whether there are any dimensions of encoding for which the hierarchical-processing hypothesis of multiple expectancies does not apply. For instance, the inhibition phenomenon may only occur when subjects generate expectancies for stimulus attributes that are separable and can be resolved independently (cf. Garner, 1974). Expectancies for integral dimensions, such as hue and brightness, may fall outside the domain of the hierarchical-processing hypothesis. Additionally, it will be illuminating to discover whether the 
inhibitory effect generalizes from perceptual ( $A$ vs. $V$ ) to semantic (letter vs. digit) classifications (cf. Lambert, 1987). If it does, then the strong implication will be that the hierarchical-processing hypothesis applies not only when attention is turned to peripherally based sensory pathways but also when it is turned to more centrally based memory pathways.

\section{References}

Cave, K. N., \& Wolfe, J. M. (1990). Modeling the role of parallel processing in visual search. Cognitive Psychology, 22, 225-271.

Eriksen, C. W., \& Schultz, D. W. (1979). Information processing in visual search: A continuous flow model and experimental results. Perception \& Psychophysics, 25, 249-263.

Fletcher, B. C., \& Rabbitt, P. M. A. (1978). The changing pattern of perceptual analytic strategies and response selection with practice in a two-choice reaction time task. Quarterly Journal of Experimental Psychology, 30, 417-427.

Garner, W. R. (1974). The processing of information and structure. Hillsdale, NJ: Erlbaum.

Garner, W. R. (1987). Location and color as cuing dimensions in contingent classification. Perception \& Psychophysics, 4J, 202-210.

Harter, M. R., Aine, C. J., \& Schroeder, C. (1982). Hemispheric differences in the neural processing of stimulus location and type: Effects of selective attention on visual evoked potentials. Neuropsychologia, 20, 421-438.

Hedge, A., \& Marsh, N. W. A. (1975). The effect of irrelevant spatial correspondence on two-choice response-time. Acta Psychologica, $39,427-439$.

Hillyard, S. A., \& Munte, T. F. (1984). Selective attention to color and locational cues: An analysis with event-related brain potentials. Perception \& Psychophysics, 36, 185-198.

Humphreys, G. W. (1981). Flexibility of attention between stimulus dimensions. Perception \& Psychophysics, 30, 291-302.

Kingstone, A. (in press). Combining expectancies. Quarterly Journal of Experimental Psychology.

Klein, R. M. (1977). Attention and visual dominance: A chronometric analysis. Journal of Experimental Psychology: Human Perception and Performance, 3, 365-378.

Klemmer, E. T. (1956). Time uncertainty in simple reaction time. Journal of Experimental Psychology, 51, 179-184.

Krueger, L. E., \& Shapiro, R. G. (1981). Intertrial effects of samedifferent judgements. Quarterly Journal of Experimental Psychology, 33A, 241-265.

Lambert, A. J. (1987). Expecting different categories at different locations and spatial selective attention. Quarterly Journal of Experimental Psychology, 39A, 61-76.

Lambert, A. J., \& Hockey, R. (1986). Selective attention and performance with a multidimensional visual display. Journal of Experimental Psychology: Human Perception and Performance, 12, 484495.

Maylor, E. A., \& Hockey, R. (1985). Inhibitory component of externally controlled covert orienting in visual space. Journal of Experimental Psychology: Human Perception and Performance, 11, 777787.
McClelland, J. L. (1979). On the time relations of mental processes: An examination of systems of processes in cascade. Psychological Review, 86, 287-330.

Neely, J. H. (1977). Semantic priming and retrieval from lexical memory: Roles of inhibitionless spreading activation and limitedcapacity attention. Journal of Experimental Psychology: General, 106, 226-254.

Neill, W. T. (1977). Inhibitory and facilitatory processes in selective attention. Journal of Experimental Psychology: Human Perception and Performance, 3, 444-450.

Posner, M. I. (1978). Chronometric explorations of mind. Hillsdale, NJ: Erlbaum.

Posner, M. I., \& Cohen, Y. (1984). Components of visual orienting. In H. Bouma \& D. Bouwhuis (Eds.), Attention and performance $X$ (pp. 531-556). London: Erlbaum.

Posner, M. I., \& Snyder, C. R. R. (1975). Facilitation and inhibition in the processing of signals. In P. M. A. Rabbitt and S. Dornic (Eds.), Attention and performance $V$ (pp. 669-682). New York: Academic Press.

Posner, M. I., Snyder, C. R. R., \& Davidson, B. J. (1980). Attention and the detection of signals. Journal of Experimental Psychology: General, 109, 160-174.

Rabbitt, P. M. A. (1966). Errors and error correction in choiceresponse tasks. Journal of Experimental Psychology, 71, 264-272.

Rabbitt, P. M. A., \& Vyas, S. M. (1974). Interference between binary classification judgements and some repetition effects in a serial choice reaction time task. Journal of Experimental Psychology, 103, 1181-1190.

Rafal, R. D., Calabresi, P. A., Brennan, C. W., \& Sciolto, T. K. (1989). Saccade preparation inhibits reorienting to recently attended locations. Journal of Experimental Psychology: Human Perception and Performance, 15, 673-685.

Stoffels, E. J., van der Molen, M. W., \& Keuss, P. J. G. (1985), Intersensory facilitation and inhibition: Immediate arousal and location effects of auditory noise on visual choice reaction time. Acta Psychologica, 58, 45-62.

Stoffels, E. J., van der Molen, M. W., \& Keuss, P. J. G. (1989). An additive factors analysis of the effect(s) of location cues associated with auditory stimuli on stages of information processing. Acta Psychologica, 70, 161-197.

Tipper, S. P. (1985). The negative priming effect: Inhibitory effects of ignored primes. Quarterly Journal of Experimental Psychology, $37 A, 571-590$.

Tipper, S. P., \& Cranston, M. (1985). Selective attention and priming: Inhibitory and facilitatory effects of ignored primes. Quarterly Journal of Experimental Psychology, 37A, 591-611.

Williams, J. A. (1966). Sequential effects in disjunctive reaction time: Implications for decision models. Journal of Experimental Psychology, 71, 665-672.
Received February 7, 1990

Revision received July 16, 1990

Accepted July 18, 1990 